\title{
Design and simulation of high-swing fully differential telescopic Op-Amp
}

\author{
Zahra Pezeshki \\ Faculty of Electrical and Robotic Engineering, Shahrood University of Technology, Shahrood, Semnan, Iran
}

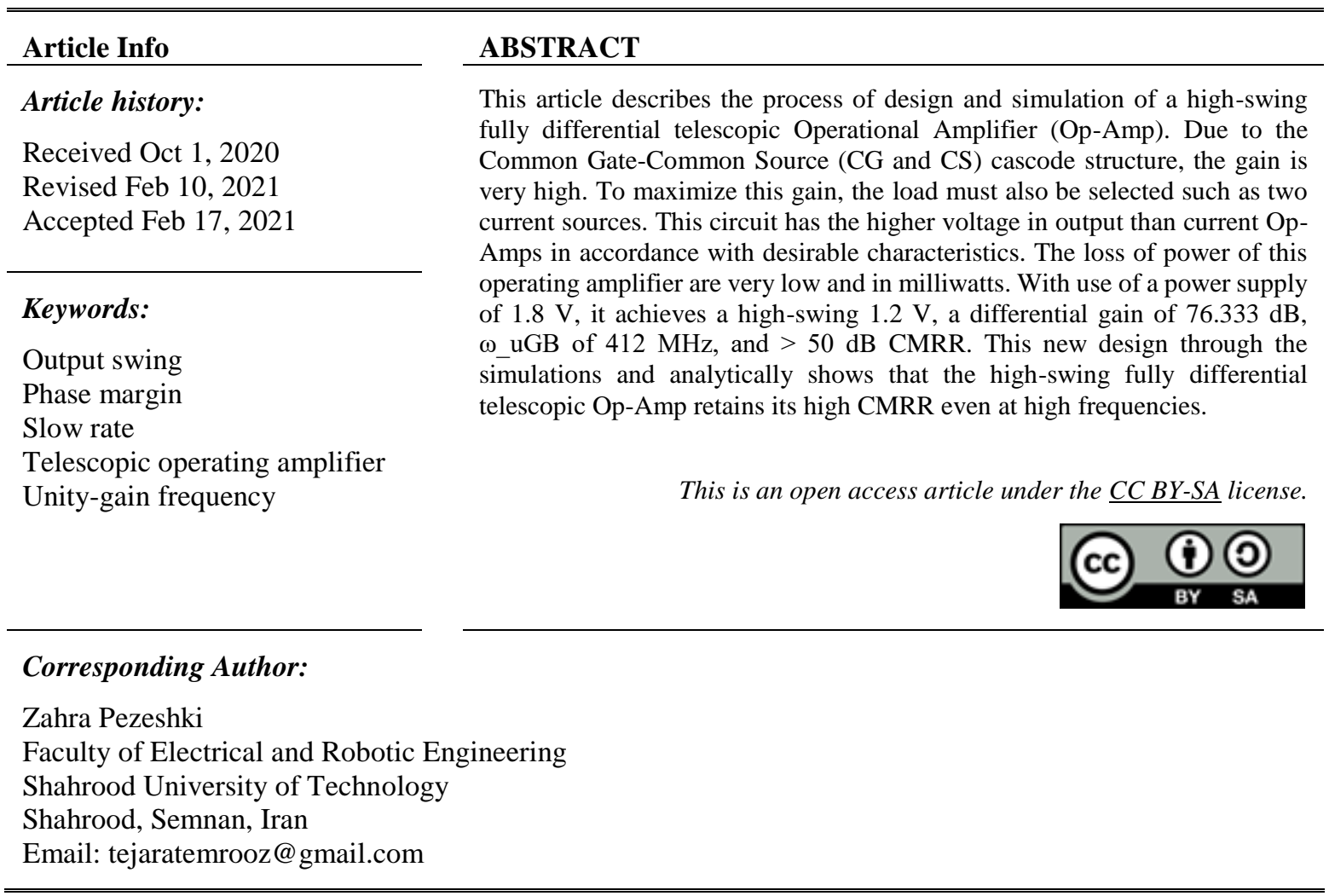

\section{INTRODUCTION}

The simplest Operational Amplifier (Op-Amp) is a high-gain differential amplifier. The amount of this gain in the range of $10^{1}-10^{5}$ is sufficient for Op-Amp applications. Op-Amp performance parameters are: open-loop gain, small signal bandwidth, large signal bandwidth, output swing, linearity, noise, offset and supply rejection [1], [2]. By designing an Op-Amp, these parameters are communicated with each other. Single cascode differential amplifiers with integrated components can hardly produce much gain. The bandwidth is determined by the load capacitance. To achieve the high gain, differential cascodes are used. These cascode connections lie between power supplies and load current sources. When the MOSFETs of each branch are aligned with each other, they create a telescopic-like structure. Therefore, this type of configuration is known as telescopic Op-Amp. The resulting circuit is symmetric with each of the output loads generated by the cascade current source. Their output swings are also limited due to the short-circuit problem of one of the inputs to the output in the applications such as source follower [3]. Another problem is power consumption. There are various designs that they have tried to reach high swing with low power consumption, but the power consumption value has not been reported [3]. In some papers, output swing is low or has not been reported too [4], [5]. The gain-boosted telescopic Op-amps can reach to high swing with low power consumption, but their design is classified into multiple design:
a. Folded cascode Op-amp [6].
b. The main Op-amp and the boosting Op-amp [7], [8].
c. The main Op-amp and the Multiplying Digital-to-Analog Converter (MDAC) architecture [9].
d. The main Op-amp and the Miller compensation [10]. 
So such a design implementation is very complicated. In this paper, author want to design a highswing fully differential telescopic Op-Amp in $0.18 \mu \mathrm{m}$ CMOS technology. All simulations of this circuit are performed in HSPICE RF software. Other softwares such as Cadence can also be used in this regard [2].

Figure 1 shows the fully differential telescopic Op-Amp. The fully differential designs include the first and secondly switched current mirrors and the output which can be a circuit duo to boosting the gain and reaching the wide band [11]-[17], but this can cause the power consumption to increase. Biasing also can improve the gain with less dissipation, e.g. Wideband QFG dynamic biasing (QFG-DB) Op-amp, but it cannot to increase the output or input voltage [18]. Some applications such as Fully Differential Difference Transconductance Amplifier (FDDTA), require low output, so the high gain is not important in their design. Because they are utilized as a low pass filter [19]. The advantage of the fully differential design is that the differential mode signal path encompasses only the n-channel MOSFETs [20]. Only NMOS transistors conduct time-varying currents, and PMOS transistors transmit a constant current. This increases the Op-Amp speed, so the mobility of the n-channel MOSFET is greater than that of the p-channel MOSFET. In my design, we use the telescopic Op-Amp [8] of Figure 1. It is a combination of Common Gate-Common Source (CS-CG) that achieves higher gain due to double load of current source.

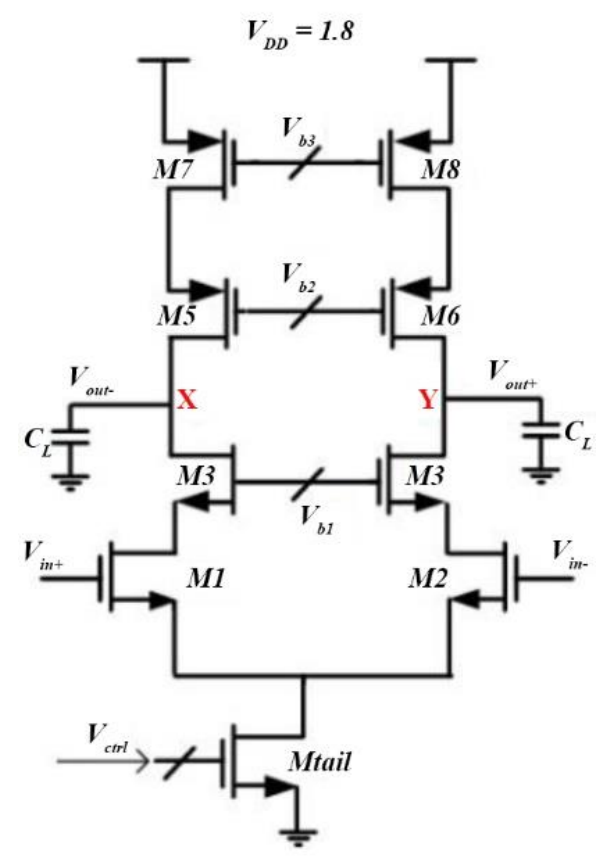

Figure 1. Telescopic cascode with NMOS inputs

Combination of CS-CG cascodes with components such as NMOS is for higher gain. Cascade load current sources with components such as PMOS are used to achieve greater drain resistance, which is used to combine CS-CG cascodes. The gain of the circuit in Figure 1 can be obtained by using half of the circuit as (1) [21].

$$
A=g_{m N}\left[\left(g_{m N} r_{O N}^{2}\right) \|\left(g_{m P} r_{O P}^{2}\right)\right]
$$

Where $g_{m}$ is the cross-conductivity and $r_{O}$ is the drain resistors in the MOSFETs. So, the gain of the telescopic cascode Op-Amp increases. The output swing is as (2):

$$
V_{c m o}=2\left[V_{D D}-\left(V_{O D 1}+V_{O D 3}+V_{O D t a i l}+\left|V_{O D 5}\right|+\left|V_{O D 7}\right|\right]\right.
$$

Where, the $V_{O D}$ is overdrive voltage of the MOSFETs, and $V_{O D \text { tail }}$ reduce through the Mtail MOSFET current source, $I_{\text {total }}$. The output voltage of this circuit is much less than that of a simple fully differential Op-Amp. So, in this paper, I want to design a new fully differential telescopic Op-amp with specifications as shown in Table1. 
Table 1. The characteristic of high-swing fully differential telescopic OP-AMP circuit

\begin{tabular}{ll}
\hline Parameter & Characteristic \\
\hline Gain & $>60 \mathrm{~dB}$ \\
CMRR & $>50 \mathrm{~dB}$ \\
$\omega_{u G B}$ & $>2 \pi \times 400 \mathrm{MHz}$ \\
Phase margin & $>60^{\circ}$ \\
Slow Rate & $>200 \mathrm{~V} / \mu \mathrm{s}$ \\
$\mathrm{V}_{\mathrm{cmi}}$ & $=1 \mathrm{~V}$ \\
$\mathrm{~V}_{\mathrm{cmo}}$ & $=1.1 \mathrm{~V}$ \\
Output Swing & $>0.5 \mathrm{~V}$ \\
Power Consumption & $>=1.2 \mathrm{~mW}$ \\
Power supply $\left(V_{D D}\right)$ & $1.8 \mathrm{~V}$ \\
\hline
\end{tabular}

\section{THE PROPOSED METHOD}

According to the Table 1, author must also consider other features of the telescopic cascade and add to the MOSFETs including $\mu_{n} C_{o x}=200 \mu A / V^{2}, \mu_{p} C_{o x}=80 \mu A / V^{2}$, body modulation effects: $\lambda_{n}=$ $0.18 \mathrm{~V}^{-1}$ and $\lambda_{p}=0.36 \mathrm{~V}^{-1}$, and the threshold voltage: $V_{T H N}=\left|V_{T H P}\right|=0.45 \mathrm{~V}$ [3], [4]. In this circuit, the total power consumption should not exceed $1.2 \mathrm{~mW}$. The current source of $V_{D D}$ is $I_{\text {tail }}=1.2 \frac{\mathrm{mW}}{1.80} \mathrm{~V}=0.67 \mathrm{~mA}$. So, each cascode branch of the Op-Amp requires $0.34 \mathrm{~mA}$ current, means $I_{\text {tail }} / 2$. On the other hand, the common mode output voltage is $1.1 \mathrm{~V}$. That is, each node $\mathrm{X}$ and $\mathrm{Y}$ in Figure 1 should be able to swing up to 1.1 volt and keep the transistors $M_{3}-M_{6}$ in saturation area. With a $1.8 \mathrm{~V}$ power supply, the total voltage available for $M_{\text {tail }}$ and each cascode branch is $0.7 \mathrm{~V}$. Thus, we have (3):

$$
\left.V_{O D 1}+V_{O D 3}+\left|V_{O D 5}\right|+\left|V_{O D 7}\right|+V_{O D t a i l}\right]=1.8-1.1=0.7
$$

Since $M_{\text {tail }}$ draws most of the current, one fifth of the voltage reaches to it, so $V_{\text {ODtail }}=0.14 \mathrm{~V}$ and $0.56 \mathrm{~V}$ remains for the four cascade transistors. The $M_{5}-M_{8}$ current sources have low mobility, so more voltage should be allocated per branch to them $(0.32 \mathrm{~V})$. Therefore, in a cascade branch, we have $V_{O D 5}+$ $V_{O D 7}=0.32 \mathrm{~V}$. The rest of the voltage is allocated to transistors $M_{1}$ and $M_{3}$. That is $V_{O D 1}+V_{O D 3}=0.24 \mathrm{~V}$. So, the overdrive voltage of transistors 5 and 7 is $0.16 \mathrm{~V}$ and the voltage of transistors 1 and 3 is $0.12 \mathrm{~V}$. The aspect ratio of $M_{1}-M_{\text {tail }}$ can be evaluated by the bias current and the overdrive voltage of each MOSFET. The relation of drain current to saturation area is (4):

$$
I_{D}=(1 / 2) \mu C_{o x}(W / L)\left(V_{G S}-V_{T H}\right)^{2}
$$

To minimize the parasitic capacitors of the integrated devices, the minimum length of each MOSFET transistor is $L=0.18 \mu \mathrm{m}$.

Then, $(W)_{1-4}=38 \mu m,(W)_{5-8}=58.25 \mu m$ and $W_{\text {tail }}=58.775 \mu m$ are obtained. So, the design was done according to the total power consumption, power supply and output swing. The amount of the gain can now be calculated from the (5),

$$
A_{v} \approx g_{m 1}\left[\left(g_{m 3} r_{o 3} r_{o 1}\right) \|\left(g_{m 5} r_{o 5} r_{o 7}\right)\right]
$$

By choosing a minimum channel length of $0.18 \mu \mathrm{m}$ for all MOSFET transistors we have $g_{m}$ and $r_{o}$ which are obtained from (6) and (7).

$$
\begin{aligned}
& g_{m}=2 I_{D} / V_{O D} \\
& r_{o}=1 / \lambda I_{D}
\end{aligned}
$$

So, author have:

$$
\begin{aligned}
& g_{m 1-4}=\frac{2 \times 0.32 \times 10^{-3}}{0.123}=5.203 \mathrm{~mA} / \mathrm{V} \\
& g_{m 5-8}=\frac{2 \times 0.32 \times 10^{-3}}{0.157}=4.764 \mathrm{~mA} / \mathrm{V} \\
& r_{o 1-4}=\frac{1}{0.18 \times 0.32 \times 10^{-3}} \approx 17361.11 \Omega
\end{aligned}
$$




$$
r_{o 5-8}=\frac{1}{0.36 \times 0.32 \times 10^{-3}} \approx 8680.56 \Omega
$$
Op-Amp.

In this paper, author propose the circuit of Figure 2, called high-swing fully differential telescopic

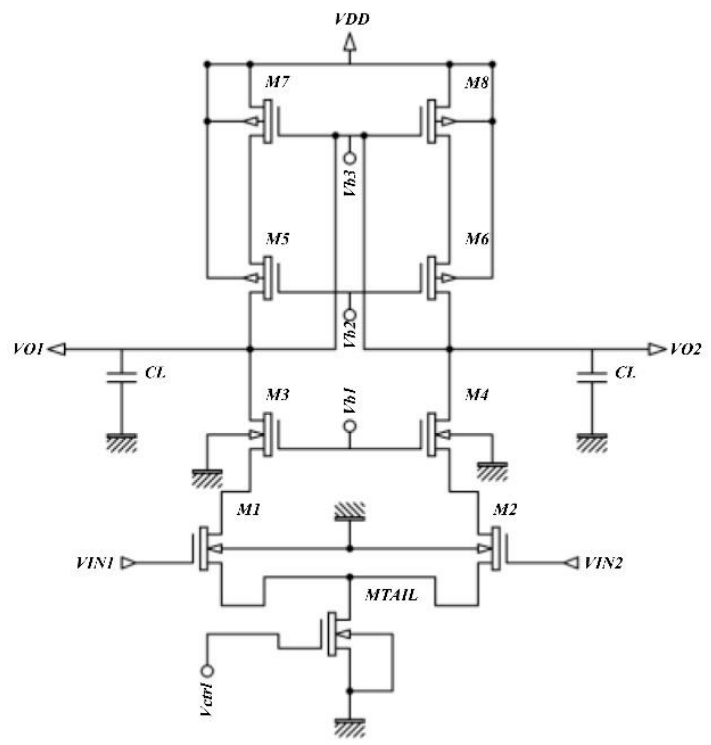

Figure 2. The proposed high-swing fully differential telescopic Op-Amp circuit

For doing simulation, firstly author must get the working point of all transistors using the ".op" command (all transistors are in saturation area) see Figure 3.

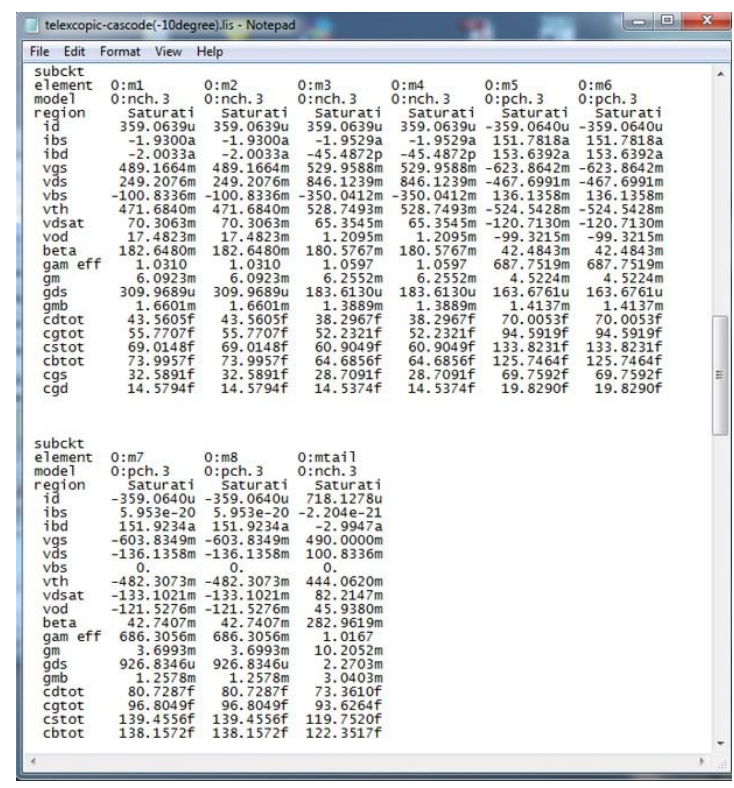

Figure 3. Working point of the proposed high-swing fully differential telescopic Op-Amp circuit

To keep the all transistors in the saturation area, the bias voltages, control voltage, and maximum and minimum input voltages, $V_{C M I}$, are calculated to drive the transistors to the saturation area as:

$$
V_{c t r l}=V_{G S t a i l} \text { if } V_{\text {ODtail }}>V_{G S t a i l}-V_{t h}
$$




$$
\begin{gathered}
V_{b 1}=V_{G S 3}+V_{O D M 1}+V_{O D t a i l} \text { if } V_{O D 3}>V_{G S 3}-V_{t h} \\
V_{b 2}=V_{D D}-V_{O D M 7}-V_{G S 5} \text { if } V_{O D 5}<V_{G S 5}-V_{t h} \\
V_{b 3}=V_{D D}-V_{G S 7} \text { if } V_{O D 7}<V_{G S 7}-V_{t h} \\
\left\{V_{\text {inMax }}=V_{D D}-2 V_{O D 7}-V_{O D 3} \text { and } V_{\text {inmin }}=V_{G S 1}+V_{O D t a i l} \text { if } V_{O D 1}>V_{G S 1}-V_{t h}\right\}
\end{gathered}
$$

So after simulation, all transistors are active and their current are regulated which the total power consumption of the circuit obtains as $1.2 \mathrm{~mW}$ see Figure 4 .

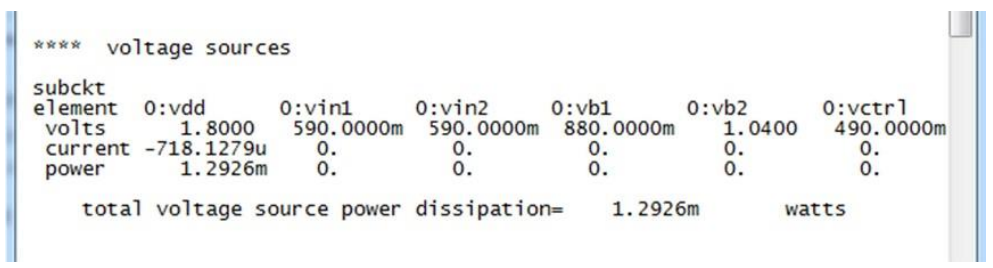

Figure 4. Power consumption of the circuit $\left(P_{\text {diss }}\right)$.

\section{RESULTS AND DISSCUSION}

In this section, author want to discuss about the simulation. According to the Figure 4 , the current drawn from the power supply is $0.72 \mathrm{~mA}$ and the total power consumption of the circuit is $1.2 \mathrm{~mW}$. The output voltage response as shown in Figure 5 is $1.2 \mathrm{~V}$.

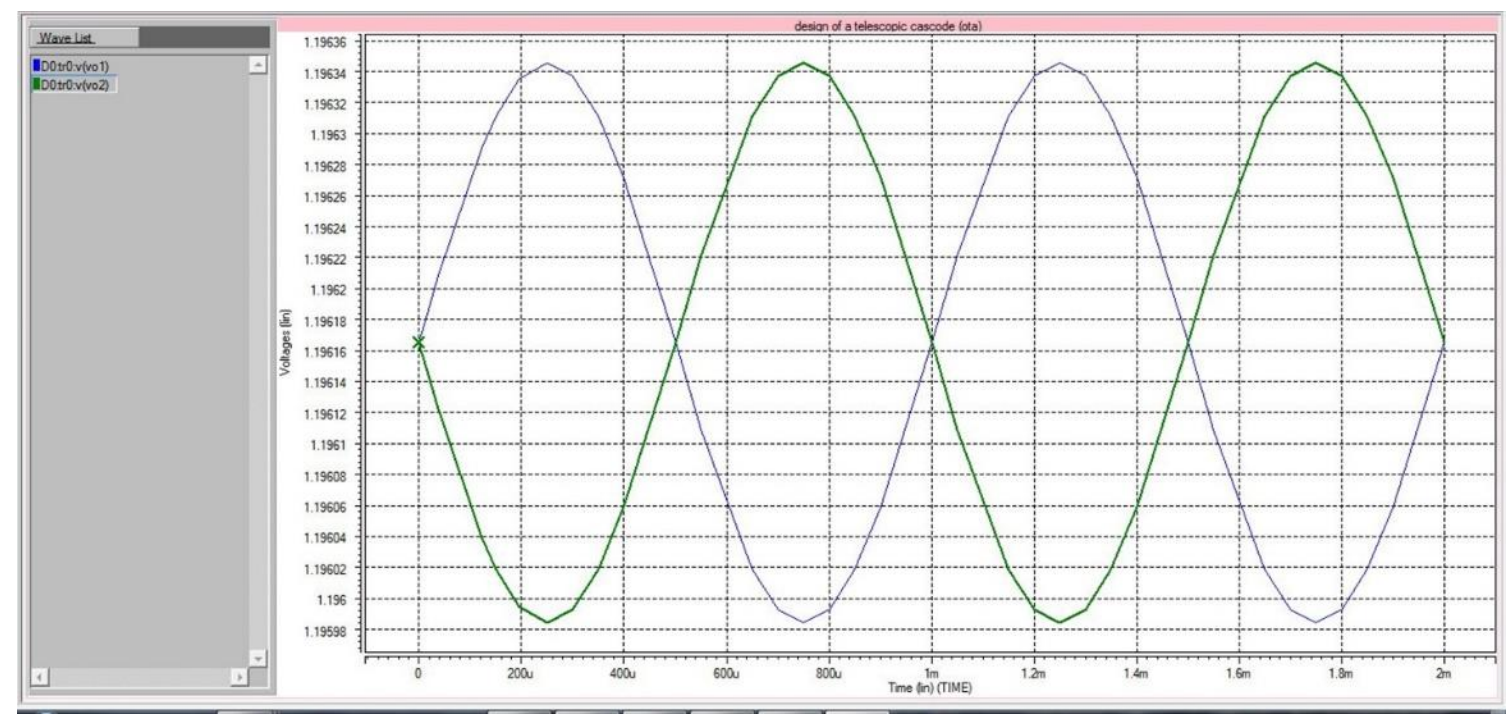

Figure 5. The output swing of the proposed high-swing fully differential telescopic Op-Amp circuit

In Figure 6, the circuit gain is greater than $60 d B$ and $76.333 d B(111.75-35.417=76.333 \mathrm{~dB})$. So the Common Mode Rejection Ratio (CMRR) is greater than $50 \mathrm{~dB}$.

At frequency of $412 \mathrm{MHz}$, the gain reaches one and thus the unity-gain frequency value, $\omega_{U G B}$, is 412 MHz see Figure 7.

According to the Figure 7, at the $\omega_{U G B}=412 \mathrm{MHz}$, the output phase value of the system is $-61.7^{\circ}$, so the Phase Margin $(\mathrm{PM})$ of the circuit is $180^{\circ} \mathrm{C}-61.7^{\circ} \mathrm{C}=118.3^{\circ} \mathrm{C}$, which a desirable value is. Further, the Total Harmonic Distortion (THD) of the circuit is $0.332 \%$ for both $V_{O 1}$ and $V_{O 2}$ outputs see Figure 8 . 


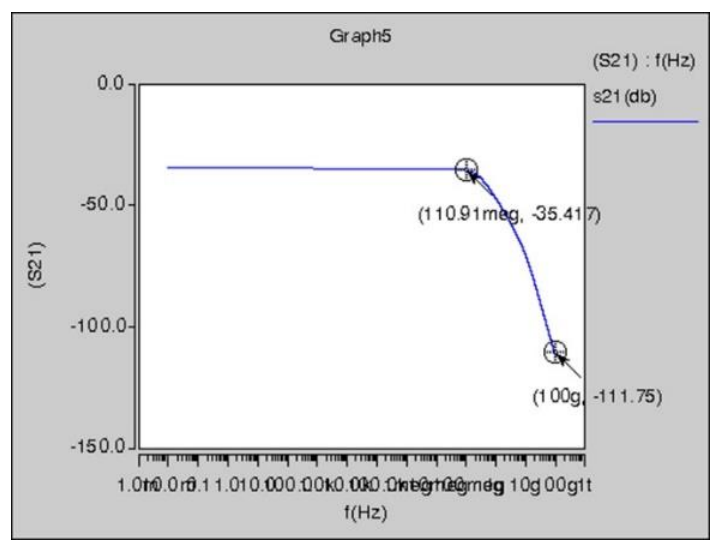

Figure 6. Frequency response of the proposed high-swing fully differential telescopic Op-Amp circuit

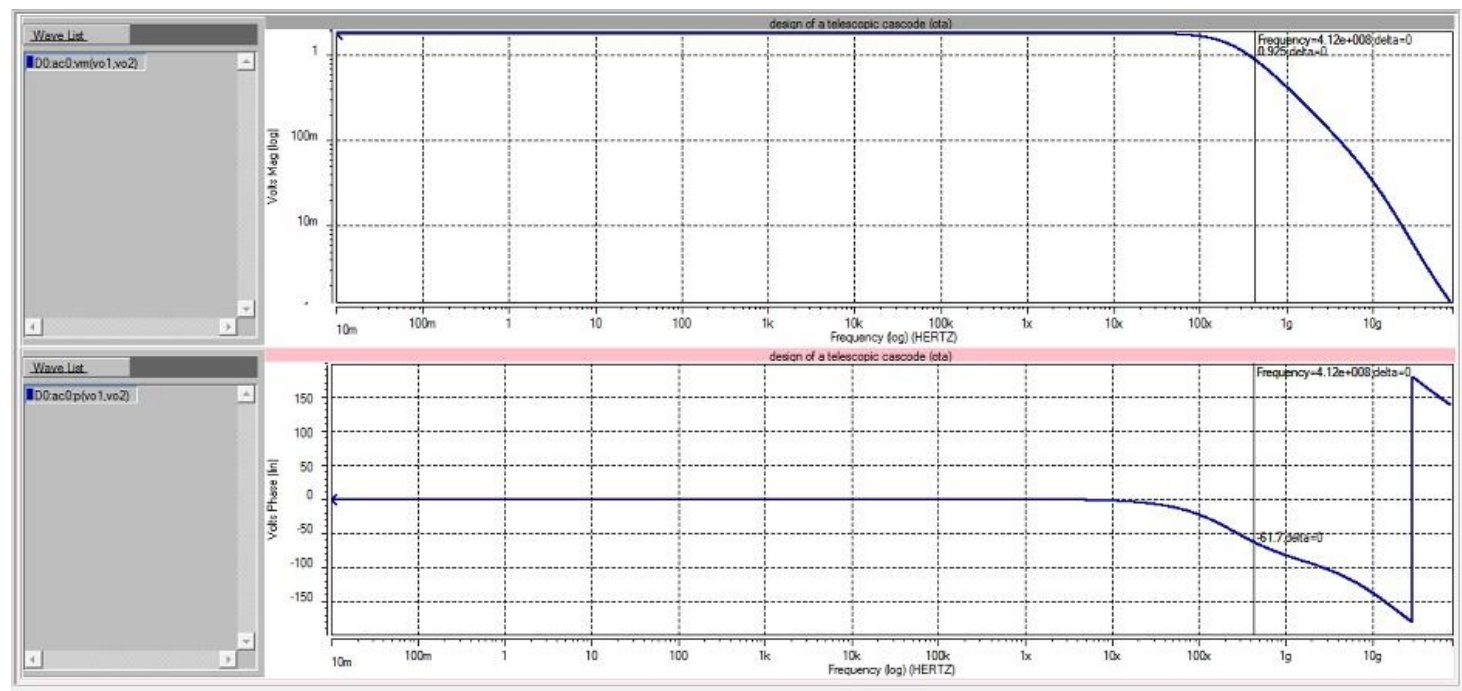

Figure 7. The $\omega_{U G B}$ and the output system phase of the proposed high-swing fully differential telescopic Op-Amp circuit

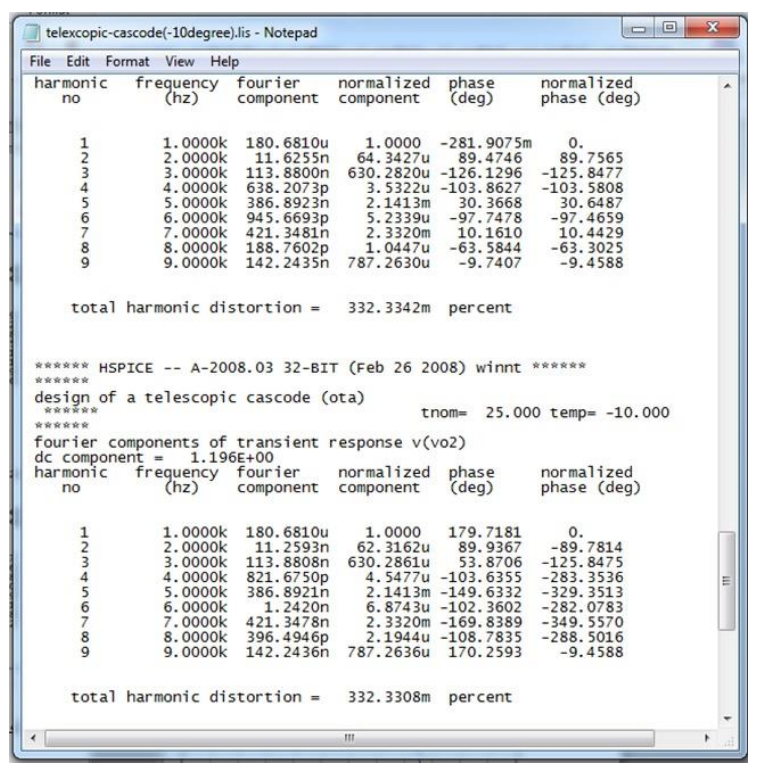

Figure 8. The THD of the proposed high-swing fully differential telescopic Op-Amp circuit 
Author can calculate the slow rate as (8) [7], [22].

$S R \equiv d V_{\text {OUt }} /\left.d t\right|_{\text {Max }}=I_{\text {tail }} / C_{L}=\left(2 I_{D M 1.2}\right) / C_{L}$

Where $I_{D M 1.2}$ is the bias current of transistors 1 and 2 . The value of the slow rate is obtained 261.25 volts per microsecond $(\mathrm{MHz})$ from the simulation see Figure 9.

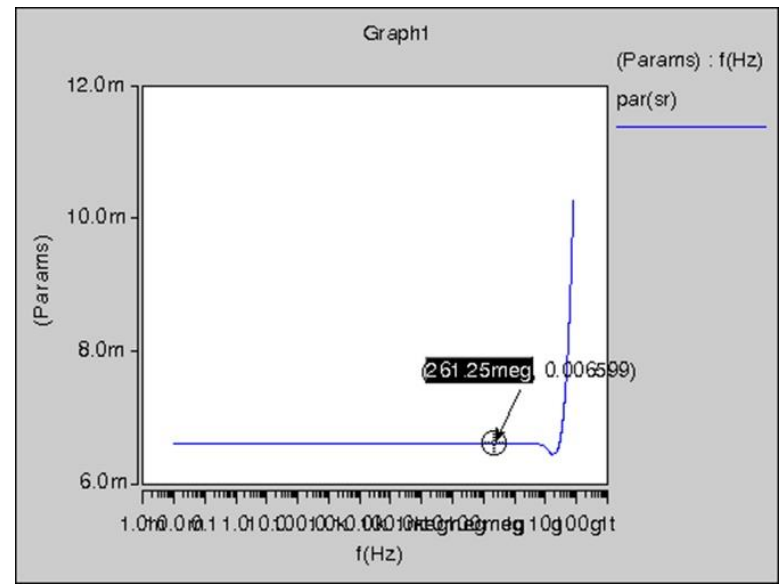

Figure 9. The slow rate of high-swing fully differential telescopic Op-Amp circuit

For noise analysis, I have to send the noise to the system, for example, with a 50 ohmic pull-up resistor (p1): $\mathrm{p} 1$ in 0 port $=1 \mathrm{ac}=0.1 \mathrm{dc}=2.1 \mathrm{z} 0=50$

The resistance value from the $V_{O 1}$ output to $V_{D D}$ is calculated $g_{m 5} r_{o 5} r_{o 7}$, which is $359 \mathrm{k} \Omega$. The output noise is $101.9337 \mu$ Volts see Figure 10.

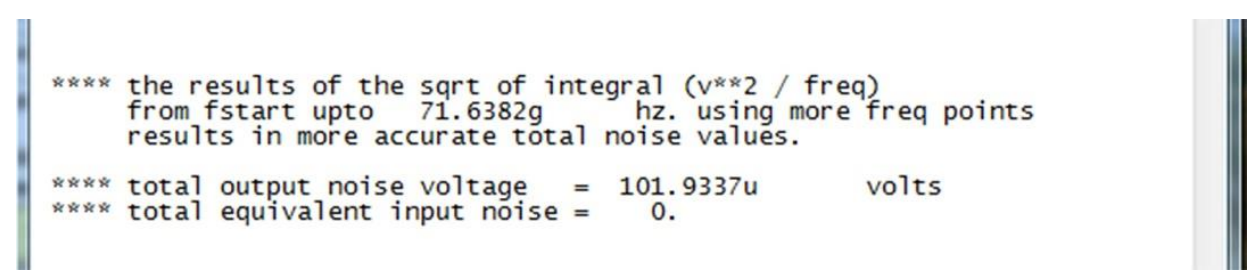

Figure 10. The output noise of the proposed high-swing fully differential telescopic Op-Amp circuit Table 2.

The characteristic table of the proposed high-swing fully differential telescopic Op-Amp circuit is as

Table 2. The Characteristic of the proposed high-swing fully differential telescopic OP-Amp circuit

\begin{tabular}{ll}
\hline Characteristic & Description \\
\hline Power supply & $1.8 \mathrm{~V}$ \\
Power consumption & $1.2 \mathrm{~mW}$ \\
Gain & $76.333 \mathrm{~dB}$ \\
$\omega_{\text {UGB }}$ & $412 \mathrm{MHz}$ \\
Output Phase Value & $-61.7^{\circ}$ \\
PM & $118.3^{\circ}$ \\
THD & $0.332 \%$ \\
Slow Rate & $261.25 \mathrm{MHz}$ \\
Total Output Noise Voltage & $101.9337 \mu \mathrm{V}$ \\
Output Swing Voltage & $1.2 \mathrm{~V}$ \\
CMRR & $>50 \mathrm{~dB}$ \\
\hline
\end{tabular}


The value of $\left(A_{v} \times \omega_{U G B}\right) / P_{\text {diss }}$ obtains as:

$$
\left(A_{v} \times \omega_{U G B}\right) / P_{\text {diss }}=\left(76.333 \times 412 \times 10^{6}\right) /\left(1.2 \times 10^{-3}\right) \cong 26207.7 \times 10^{9} \mathrm{~dB} \cdot \mathrm{Hz} / \mathrm{Watt}
$$

The characteristic of this proposed Op-amp is good comparing the other Op-amps. Table 3 compares the characteristic of this Op-amp with other designs.

Table 3. Comparison the Characteristic of the proposed high-swing fully differential telescopic OP-Amp circuit with other circuits

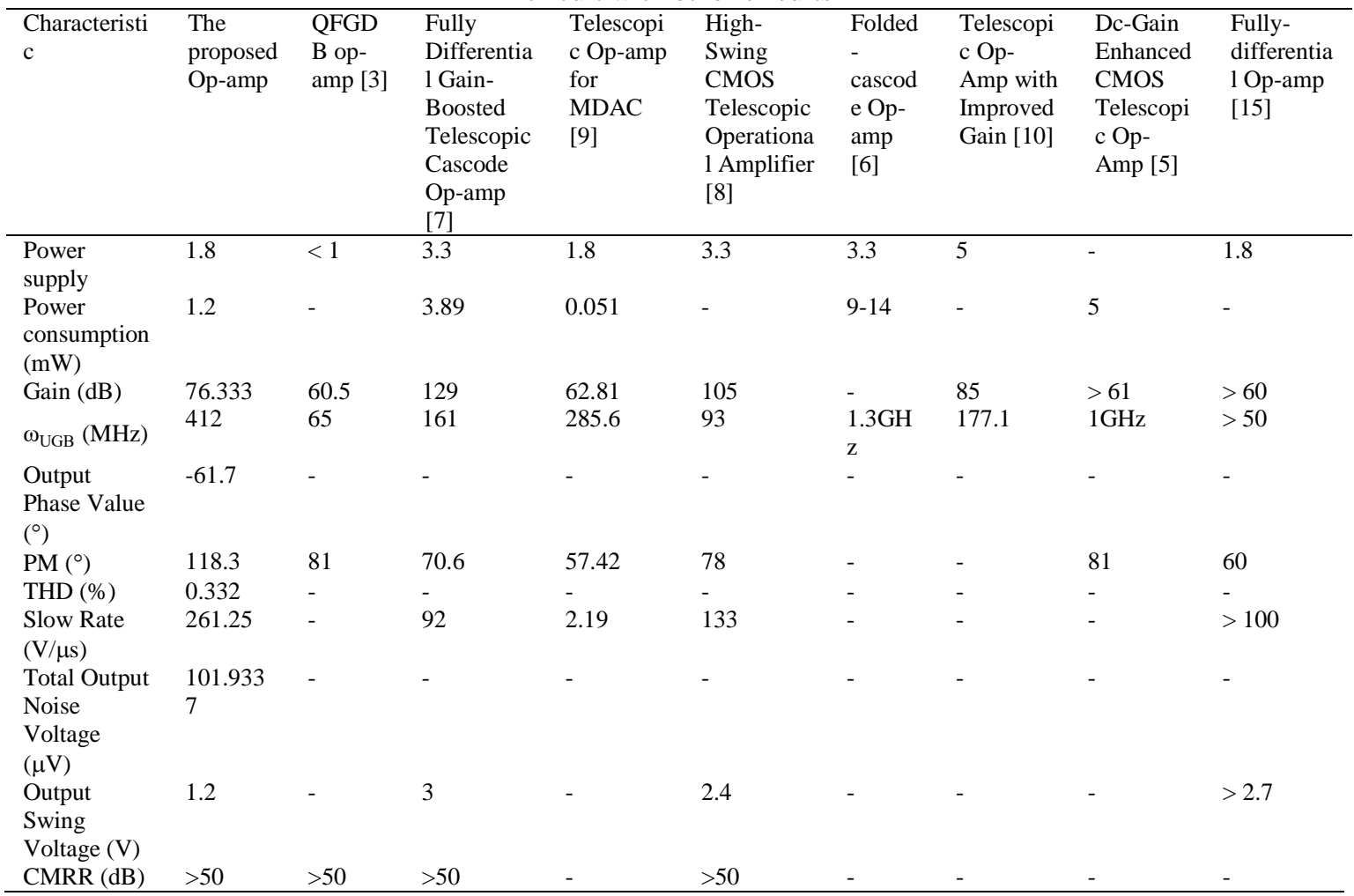

\section{CONCLUSION}

This paper presents the design of an of high-swing fully differential telescopic Op-Amp circuit with 0.18 CMOS technology. The results of the circuit simulation with HSPICE RF software show that the circuit parameters have acceptable values. One of the important features that this paper wants to acquire is higher voltage than current Op-Amps in accordance with desirable parameters. Finally, by compromising the circuit parameters, we were able to obtain a good performance from the circuit design. As the amplification of signals in many electronic circuits plays a key role and can be easily implemented with Op-Amp, this design can demonstrate this reality which can implemented in a Very Large-Scale Integration (VLSI) chip.

\section{ACKNOWLEDGEMENTS}

This paper is a technical report related to the Linear IC course which has been performed in Shahrood University of Technology, Semnan, Iran.

\section{REFERENCES}

[1] A. Worapishet, A. Demosthenous and X. Liu, "A CMOS Instrumentation Amplifier With 90-dB CMRR at 2-MHz Using Capacitive Neutralization: Analysis, Design Considerations, and Implementation," in IEEE Transactions on Circuits and Systems I: Regular Papers, vol. 58, no. 4, pp. 699-710, April 2011, doi: 10.1109/TCSI.2010.2078850.

[2] H. Qiao, "Design of a CMOS Two-stage Fully Differential Operation Amplifier," J. Phys.: Conf. Ser. vol. 1449, no. 2020, pp. 012084, 2020. 
[3] J. Ramirez-Angulo, M. Sawant, A. Lopez-Martin, and R.G. Carvajal, "A power efficient and simple scheme for dynamically biasing cascode amplifiers and telescopic op-amps," integration, the VLSI journal, vol. 41, no. 4, pp. 539-543, 2008.

[4] D.S. Shylu1, D. Jackuline Moni, and N. Brigit Francis, "Design and Analysis of Low Power Pseudo Differential Class-Ab Telescopic Operational Amplifier," International Journal of Research in Engineering and Technology, vol. 3, no. 19 , pp. 234-238, 2014.

[5] B. Alizadeh, Sadegh Mohammadzadeh Bazarchi, and A. Dadashi, "Dc-gain enhanced CMOS Telescopic op-amp, “2011 Faible Tension Faible Consommation (FTFC)," Marrakech, pp. 91-94, 2011.

[6] A. Baradaran Rezaeii, K. Dadashi, and S. Mahdavi, "Gain-Bandwidth Enhancement in Folded-Cascode Op-Amp, "2016 1st International Conference on New Research Achievements in Electrical and Computer Engineering," 2016. [Online] https://www.civilica.com/Paper-CBCONF01-CBCONF01_0070.html.

[7] Wang Jin and Qiu Yulin, "Analysis and design of fully differential gain-boosted telescopic cascode Op-Amp, "Proceedings. 7th International Conference on Solid-State and Integrated Circuits Technology," Beijing, China, vol. 2, pp. 1457-1460, 2004, doi: 10.1109/ICSICT.2004.1436874.

[8] K. Gulati and Hae-Seung Lee, "A high-swing CMOS telescopic operational amplifier," in IEEE Journal of SolidState Circuits, vol. 33, no. 12, pp. 2010-2019, Dec. 1998, doi: 10.1109/4.735542.

[9] A. Dendouga and S. Oussalah, "Telescopic Op-Amp Optimization for MDAC Circuit Design," Electronics, vol. 20, no. 2, pp. 55-61, 2016.

[10] M. Suthar, N. Gupta, and P. Soni, "Proposed Telescopic Op-Amp with Improved Gain," International Journal of Scientific and Research Publications, vol. 2, no. 2, pp. 1-3, 2012.

[11] G.L. Schaffer and C.A. Cupertino, "Fully differential autozeroing amplifier," United States Patent, US 8.487.697 B2, 2013.

[12] S. Chakraborty, "Fully Differential Wideband Amplifier," NM6007 Analog IC Laboratory Course, NTU-MSc in Integrated Circuit Design, Technische Univeritat Munchen, Nanyang Technological University, Technical report, 2017, doi: 10.13140/RG.2.2.28346.08645.

[13] N. Hossein-Zadeh, M. Daliri, S. Magierowski and E. Ghafar-Zadeh, "A Novel Fully Differential NMR Transciever," 2018 IEEE Life Sciences Conference (LSC), Montreal, QC, pp. 13-16, 2018, doi: 10.1109/LSC.2018.8572220.

[14] J. Huijsing, "Fully Differential Operational Amplifiers," In: Operational Amplifiers. Springer, Cham, pp. 289-306, 2017, doi: 10.1007/978-3-319-28127-8_8.

[15] E. Balti, "Fully-differential opamp design," University of Idaho, Department of Electrical and Computer Engineering, Technical Report, ECE-515 Analog Integrated Circuit Design, Sep 2018.

[16] R.J. Baker, “Fully-Differential Amplifier,” United States Patent, US 5.953.276, 1999.

[17] A. Fant, L.Sant, and P. Vernei Torta, "Input Common Mode Circuit for A Fully Differental Amplifer," United States Patent, US 8.633,765 B2, 2014.

[18] J. Ramirez-Angulo, M. S. Sawant, R. G. Carvajal and A. J. Lopez-Martin, "New compact and power efficient dynamically biased cascode mirrors and telescopic op-amps," 2006 IEEE International Symposium on Circuits and Systems, Island of Kos, 2006, pp.2833-2836, doi: 10.1109/ISCAS.2006.1693214.

[19] F. Khateb, M. Kumngern, T. Kulej, and V. Kledrowetz, "Low-voltage fully differential difference transconductance amplifier," IET Circuits Devices Syst., vol. 12, no. 1, pp. 73-81, 2018.

[20] A.S. Sedra, and K.C. Smith, Microelectronic circuits theory and applications, Oxford University press, 7th edition, pp. 848-857, 2010.

[21] B. Razavi, Design of analog CMOS Integrated Circuits, Tata Megraw Hill, 2002 edition, pp. 291-307.

[22] T.C. Carusone D. Johns and K. Martin, Analog Integrated Circuit Design, Wiley, 2nd Ed., 2011, pp. 1-816.

\section{BIOGRAPHIES OF AUTHOR}

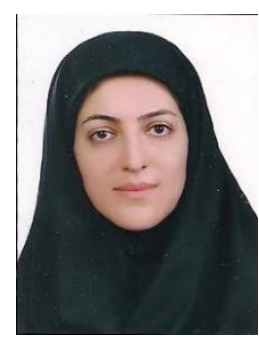

Zahra Pezeshki is a Master holder in Electronic Integrated Circuits. She's worked on electronic circuit design and project management from 2002 to now as well as obtained one patent in 2007 for solving irrigation problems. Furthermore, she has cooperated with Iran Construction Engineering Organization (IRCEO) as a researcher since 2012 whereas she works on developing new BIM standards for construction industry. Currently, she works as an engineer, senior lecturer and a journalist working with IRCEO and Iranian press. She is greatly interested in energy issues, sustainability and optimization. Her research activities are highly in design, modeling, programming and manufacturing of electrical maps, electrical boards and AI devices to increase thermal comfort and save energy. 